\title{
INTERPOLATING WAVELETS ON THE SPHERE ${ }^{1}$
}

\author{
Nikolai I. Chernykh \\ Krasovskii Institute of Mathematics and Mechanics, \\ Ural Branch of the Russian Academy of Sciences, \\ 16 S. Kovalevskaya Str., Ekaterinburg, 620990, Russia \\ Ural Federal University, \\ 51 Lenin aven., Ekaterinburg, 620000, Russia \\ chernykh@imm.uran.ru
}

\begin{abstract}
There are several works where bases of wavelets on the sphere (mainly orthogonal and wavelet-like bases) were constructed. In all such constructions, the authors seek to preserve the most important properties of classical wavelets including constructions on the basis of the lifting-scheme. In the present paper, we propose one more construction of wavelets on the sphere. Although two of three systems of wavelets constructed in this paper are orthogonal, we are more interested in their interpolation properties. Our main idea consists in a special double expansion of the unit sphere in $\mathbb{R}^{3}$ such that any continuous function on this sphere defined in spherical coordinates is easily mapped into a $2 \pi$-periodic function on the plane. After that everything becomes simple, since the classical scheme of the tensor product of one-dimensional bases of functional spaces works to construct bases of spaces of functions of several variables.

Keywords: Wavelets, Multiresolution analysis, Scaling functions, Interpolating wavelets, Best approximation, Trigonometric polynomials.
\end{abstract}

\section{Introduction}

Different systems of wavelets on the sphere are constructed and studied in a number of works. We would like to note the constructions in the paper by Skopina [9]. They are beautiful but difficult to put in practice, as their author notes herself. In [2], the ideas of these constructions were extended to spheres in $\mathbb{R}^{n}$. These and some other works mentioned below contain a good analysis of the studies on the specified or close subject. In $[4,6,7]$, to construct bases of wavelets on spheres in $\mathbb{S}^{2}$ and $\mathbb{S}^{3}$, the tensor product of bases of one-dimensional wavelets is used including a basis of exponential splines on a segment. In the papers $[1,5,8]$, which contain much of the bibliography related or close to the subject and the analysis of the previous results, in particular, the liftingscheme technique is used to construct biorthogonal wavelets on the sphere. This is accompanied by rejecting a number of properties of classical wavelets including, for instance, shifts with a constant step at each scaling level and with localization of the compression-stretching operation in the right places. In the present paper, we attempt to preserve the standard properties of classical wavelets on the line and on the period in the construction of wavelets on the sphere. In so doing, we give preference to interpolating wavelets. Orthogonal wavelets are only defined. The study of their approximative properties is postponed for the future. Here, for the classical schemes to construct wavelets on the sphere to work, we carry out a double expansion of the unit sphere with a special extension to it of the function originally defined on the sphere. This makes it possible to apply onedimensional periodic interpolation and interpolation-orthogonal bases of expanding subspaces of

\footnotetext{
${ }^{1}$ This work was supported by the Russian Academic Excellence Project (agreement no. 02.A03.21.0006 of August 27, 2013, between the Ministry of Education and Science of the Russian Federation and Ural Federal University).
} 
multiresolution analysis (constructed and studied in $[3,10]$ ) to construct wavelets on the expanded sphere.

\section{Construction of wavelets on the sphere}

Without loss of generality, we assume that $\mathbb{S}$ is the sphere of unit radius centered at the origin of a Cartesian coordinate system, $(\theta, \varphi)$ are spherical coordinates of points of $\mathbb{S}\left(\theta \uparrow_{0}^{\pi}\right.$ denotes latitude and $\varphi \uparrow_{0}^{2 \pi}$ denotes longitude) associated in a standard way with the Cartesian coordinates. Thus,

$$
\mathbb{S}=\{M(\theta, \varphi) \in \mathbb{S}: 0 \leq \theta \leq \pi, 0 \leq \varphi<2 \pi\}
$$

For a uniform grid with any small step $h=2 \pi / l(l \in \mathbb{N}, l \gg 1)$ in the angular coordinates $\theta, \varphi$, the geometrical sizes of cells of the corresponding grids on the sphere are strongly nonuniform. We have cells with size of order $h \times h$ in $\mathbb{R}^{3}$ near the equator, where $\theta$ is close to $\pi / 2$, and we have cells with size of order $h \times h^{2}$ near the poles, where $\theta$ is close to 0 in the case of the north pole $N$ or $\theta$ is close to $\pi$ in the case of the south pole $S$. On $\mathbb{S}$, every value $\varphi \in \mathbb{T}$ (where $\varphi$ and $\varphi_{ \pm 2 l \pi}$ are indistinguishable) determines the $\varphi$-meridian, i.e., the great circle arc

$$
M_{\varphi}:=\{M(\theta, \varphi): 0 \leq \theta \leq \pi\}
$$

and every value $\theta \in(0, \pi)$ determines $\theta$-latitude, i.e., the circle $M^{\theta}:=\{M(\theta, \varphi): 0 \leq \varphi<2 \pi\}$ of radius $r_{\theta}=\sin \theta$ in the plane $z_{\theta}=\cos \theta$ centered at the point $\left(0,0, z_{\theta}\right)$ of the Cartesian system. Despite the noted disadvantage of spherical coordinates and the specified grids on $\mathbb{S}$ uniform in $\theta$ and $\varphi$, their application is profitable and simple for both the construction of wavelets on $\mathbb{S}$ and the practical use of the wavelets in computational algorithms.

Thus, to construct basis scaling functions of the subspaces $V_{j}(\mathbb{S}) \subset L^{2}(\mathbb{S})\left(j \in \mathbb{Z}_{+}\right)$of multiresolu-tion analysis on $\mathbb{S}$, a usual method of passage from one-dimensional to multi-dimensional wavelets can be used here by choosing as those the tensor product of the bases of the subspaces $V_{j}(\mathbb{T})$ of the space $L^{2}(\mathbb{T})$ of $2 \pi$-periodic functions and the bases of the subspaces $V_{j}[0, \pi] \subset L^{2}[0, \pi]$ as done in [4]. It is true that, in this case, one has to use a construction of wavelets on a segment, which is more complicated than that on the line or on a period, for instance, applying a "folding" operation. Instead of this, in the present paper, the sphere "doubles". Due to this, the construction of bases of the subspaces $V_{j}(\mathbb{S})$ reduces to the tensor product of two (possibly different) bases of the subspaces $V_{j}(\mathbb{T})$ in the variables $\varphi$ and $\theta$, respectively.

It is clear that any $\varphi$-meridian $M_{\varphi}$ is connected with the opposite $(\varphi \pm \pi)$-meridian $M_{\varphi \pm \pi}$ on which as well as on $M_{\varphi}$, by the definition of the spherical coordinates $(\theta, \varphi), \theta$ changes from 0 (at the pole $N$ ) to $\pi$ (at the pole $S$ ). These two meridians form together the great circle $C_{\varphi}$ on $\mathbb{S}$. Keeping the bypass direction of the $\varphi$-meridian by the points $M(\theta, \varphi)$ when $\theta$ increases on $\varphi$-meridian and changing it to the opposite on the $\varphi \pm \pi$-meridian, for every $\varphi \in[0,2 \pi]$, we define the full $\varphi$-meridian as follows:

$$
C_{\varphi}:=\left\{M(\theta, \varphi) \in M_{\varphi} \cup M_{\varphi \pm \pi}: 0 \leq \theta \leq 2 \pi\right\},
$$

where $M(0, \varphi)=M(2 \pi, \varphi)$.

We note that, although the full $\varphi$-meridian $C_{\varphi}$, element-wisely coinciding with $M_{\varphi} \cup M_{\varphi \pm \pi}$, crosses the equator $\{M(\theta, \varphi): \theta=\pi / 2,0 \leq \varphi<2 \pi\}$ in the two points $(\pi / 2, \varphi)$ and $(\pi / 2, \varphi \pm \pi)$, this meridian is completely determined by the value of the angle $\varphi$, since its bypass direction with the increase of $\theta$ is determined by the movement direction of the point $M(\theta, \varphi)$ along the $\varphi$-meridian and is continuously extended to the $(\varphi \pm \pi)$-meridian changing its original direction from $N$ to $S$ to the opposite. 
Any function $f$ defined on $\mathbb{S}$ uniquely determines the function $f(\theta, \varphi)$ of the variables $\varphi \in[0,2 \pi]$ and $\theta \in[0, \pi]$. In particular, this function is uniquely defined on any full $\varphi$-meridian as a function of $\theta$, and to apply periodic wavelets in the construction of wavelets on $\mathbb{S}$, it is very important that the coordinate $\theta$ changes on $C_{\varphi}$ over the full period from 0 to $2 \pi$, since, in $C_{\varphi}$, the function $f(\theta, \varphi)$ is $2 \pi$-periodic in $\theta$ because the functions $f(0, \varphi)$ and $f(\pi, \varphi)$ on $\mathbb{S}$ do not depend on $\varphi$. However, it is easy to see that $C_{\varphi}$ and $C_{\varphi \pm \pi}$ coincide as sets of points on $\mathbb{S}$ differing only in the direction of movement of their points $M$ with coordinate $\theta$ as $\theta \uparrow_{0}^{2 \pi}$. As a result, every function $f(\theta, \varphi)$ singlevalued on $\mathbb{S}$ generates a two-valued function $F(\theta, \varphi)$ of the variable $\theta$ on every set $C_{\varphi}=C_{\varphi \pm \pi}$ and, hence, on $\mathbb{S}$. Namely, for any $\varphi \in[0,2 \pi)$, we have

$$
F(\theta, \varphi)=\left\{\begin{array}{cll}
f(\theta, \varphi), \theta \uparrow_{0}^{\pi} & \text { on } & M_{\varphi}, \\
f(2 \pi-\theta, \varphi \pm \pi), \theta \uparrow_{\pi}^{2 \pi} & \text { on } & M_{\varphi \pm \pi}
\end{array}\right.
$$

on $C_{\varphi}$ and

$$
F(\theta, \varphi)=\left\{\begin{array}{cll}
f(\theta, \varphi \pm \pi), \theta \uparrow_{0}^{\pi} & \text { on } M_{\varphi \pm \pi}, \\
f(2 \pi-\theta, \varphi), \theta \uparrow_{\pi}^{2 \pi} & \text { on } M_{\varphi}
\end{array}\right.
$$

on $C_{\varphi \pm \pi}$. Obviously, this function completely restores $f(\theta, \varphi)$ already for $0 \leq \varphi<\pi$. However, it is also important for us to preserve the $2 \pi$-periodicity of the function $F$ in $\varphi$.

To avoid the two-valuedness, we use the fact that $\mathbb{S}$ is a two-sided surface and we distinguish external and internal points $M(r, \theta, \varphi)$ of $\mathbb{S}$ considering them as if for $r=1+0$ and $r=1-0$.

In what follows, we assume that the continuous passage from one side of $\mathbb{S}$ to the other is allowed only through the poles $N$ and $S$ of the sphere $\mathbb{S}$. In so doing, any full $\varphi$-meridian is not placed on one side of $\mathbb{S}$ but is placed in two parts on different sides of $\mathbb{S}$. We place the part $M_{\varphi}$ of any full $\varphi$-meridian $C_{\varphi}$ on the external side $\mathbb{S}_{1+0}$ of $\mathbb{S}$ and the part $M_{\varphi \pm \pi}$ with $\theta \uparrow_{\pi}^{2 \pi}$ on the internal part $\mathbb{S}_{1-0}$ of $\mathbb{S}$. As a result, the function $F(\theta, \varphi)$ in (1.1) becomes a single-valued and well-defined function on $S_{1+0} \cup S_{1-0}$ coinciding with $f(\theta, \varphi)$ on $\mathbb{S}_{1+0}$. On the internal side $\mathbb{S}_{1-0}$, the function $F(\theta, \varphi)$ is defined by the part of formula (1.1) which relates to the $(\varphi \pm \pi)$-meridian. Formula (1.2) is given only to explain the reason of the two-valuedness of the function $F(\theta, \varphi)$ on $\mathbb{S}$.

Now, according the usual classical Meyer scheme, one can easily construct a multiresolution analysis on the double sphere $\widetilde{\mathbb{S}}_{(2)}=S_{1+0} \cup S_{1-0}$ with angular coordinates of points on $\widetilde{\mathbb{S}}_{(2)}$ still denoted by $(\theta, \varphi)$. In this case, $\theta$ changes from 0 to $2 \pi$ on any full $\varphi$-meridian and values of $\varphi$ can still be bounded by the interval $[0,2 \pi)$. The coordinates of points $M(\theta, \varphi)$ on $\mathbb{S}_{1+0}$ are usual spherical coordinates. They are extended on $\mathbb{S}_{1-0}$ as follows: the $\varphi$-coordinate of the point $M \in \mathbb{S}_{1-0}$ coincides with its value in the original spherical coordinate system, and the value of its usual spherical latitude, say $\tau$, is replaced by $\theta=2 \pi-\tau$. It is easy to see that the point $M$ with such coordinates $(\theta, \varphi)$ belongs to the part of the full $(\varphi \pm \pi)$-meridian lying on $\mathbb{S}_{1-0}$ (the sign, plus or minus, in the expression $\varphi \pm \pi$ can always be taken so that $\varphi \pm \pi \in[0,2 \pi))$.

As basic functions of the subspaces $V_{j}(\mathbb{T})$ of multiresolution analysis on $\widetilde{\mathbb{S}}_{(2)}$ (defining $V_{j}(\mathbb{T})$ themselves), we take systems of $2 \pi$-periodic functions constructed on the basis of Meyer wavelets. These are the trigonometric polynomials $\Phi_{s}^{j, k}(x)(s=1,2,3)$ generating the finite-dimensional subspaces $V_{j}(\mathbb{T})$. We use them because of their simplicity. Furthermore, in order not to calculate integral coefficients of function expansions in orthogonal systems, we restrict ourselves to the use only of the interpolation properties of multiresolution analysis on finite grides in $\theta$ and $\varphi$. Since the convergence of interpolation expansions for continuous (and especially smooth) functions on $\mathbb{S}$ occurs with high rate, there is no need to apply the subspaces $V_{j}\left(\widetilde{\mathbb{S}}_{(2)}\right)$ with large indices $j$ for practical problems. Thus, one may not be afraid of a significant concentration of grid points near the poles (especially in the case of computer implementation of algorithms of approximation of functions $f$ on $\mathbb{S}$ ). The orthogonal properties of bases can be useful when approximating functions integrable only on $\mathbb{S}$. 
Thus, in what follows, we use (see $[3,10])$ the scaling functions of periodic multiresolution analyzes:

$$
\Phi_{s}^{j, k}(x)=2^{-j} \sum_{\left|\nu / 2^{j}\right|<(1+\varepsilon) / 2} \widehat{\varphi}_{s}\left(\frac{\nu}{2^{j}}\right) e^{i \nu\left(x-2 \pi k / 2^{j}\right)}, \quad k=\overline{0,2^{j}-1}, \quad j \in \mathbb{Z}_{+}, \quad s=1,2,3,
$$

where

$$
\widehat{\varphi}_{s}(\omega)=\widehat{\varphi}_{\varepsilon}(\omega)^{2}+\left(1-\delta_{3, s}\right) i(\operatorname{sign} \omega) \widehat{\varphi}_{\varepsilon}(\omega)\left(\widehat{\varphi}_{\varepsilon}(\omega-1)+\widehat{\varphi}_{\varepsilon}(\omega+1)\right), \quad s=2,3 .
$$

In turn, $\widehat{\varphi}_{\varepsilon}(\omega), \varepsilon>0$, is an even continuous real function on $\mathbb{R}$ of Meyer type supported on the interval $|\omega|<(1+\varepsilon) / 2$ and such that $\widehat{\varphi}_{\varepsilon}(\omega)=1$ for $|\omega| \leq(1-\varepsilon) / 2(0<\varepsilon \leq 1 / 3)$, the derivative $\widehat{\varphi}_{\varepsilon}^{\prime}(\omega)$ is a function of bounded variation, and $\widehat{\varphi}_{\varepsilon}^{2}(\omega)+\widehat{\varphi}_{\varepsilon}^{2}(\omega-1)=1$ for $(1-\varepsilon) / 2<\omega \leq(1+\varepsilon) / 2$. When $s=1$, we replace $\widehat{\varphi}_{\varepsilon}(\omega)$ in (1.4) by

$$
\widehat{\varphi}_{1, \varepsilon}(\omega)=\frac{1}{\sqrt{2}} \sqrt{1+\widehat{\varphi}_{\varepsilon}(\omega)-\widehat{\varphi}_{\varepsilon}(\omega-1)-\widehat{\varphi}_{\varepsilon}(\omega+1)} .
$$

For each $s=1,2,3$, the functions $\Phi_{s}^{j, k}(x)$ form the interpolation basis of the subspaces $V_{s}^{j}(\mathbb{T})$ $\left(j \in \mathbb{Z}_{+}\right)$of $2 \pi$-periodic multiresolution analysis:

$$
\Phi_{s}^{j, k}\left(\frac{2 \pi l}{2^{j}}\right)=\delta_{k, l} \quad\left(k, l=\overline{0,2^{j}-1}\right) .
$$

In addition, for $s=1,2$ and for any $j \in \mathbb{Z}_{+}$, the system $\left\{2^{j / 2} \Phi_{s}^{j, k}(x)\right\}$ is orthonormal on $\mathbb{T}$ :

$$
\frac{1}{2 \pi} \int_{0}^{2 \pi} 2^{j} \Phi_{s}^{j, k}(x) \overline{\Phi_{s}^{j, l}(x)} d x=\delta_{k, l} \quad\left(k, l=\overline{0,2^{j}-1}\right) .
$$

For any $j$ and for $k, l=0,1, \ldots, 2^{j}-1$, we define

$$
\Phi_{s}^{j, k, l}(\theta, \varphi)=\Phi_{s}^{j, k}(\theta) \Phi_{s}^{j, l}(\varphi) \text { for }(\theta, \varphi) \in \mathbb{T} \times \mathbb{T} .
$$

Naturally, without any additional assumptions except for the $2 \pi$-periodicity, this is an interpolation system of functions on the grid $\left\{\left(2 \pi m / 2^{j}, 2 \pi n / 2^{j}\right): m, n=\overline{0,2^{j}-1}\right\}$ :

$$
\Phi_{s}^{j, k, l}\left(\frac{2 \pi m}{2^{j}}, \frac{2 \pi n}{2^{j}}\right)=\delta_{k, m} \cdot \delta_{l, n} .
$$

This system inherits in $C(\mathbb{T} \times \mathbb{T})$ all approximative properties of system (1.3) in $C[0,2 \pi]$.

\section{Approximation by interpolating wavelets in $C(\mathbb{T} \times \mathbb{T})$}

We denote by ${ }_{s} V_{j}\left(\mathbb{T}^{2}\right)$ the subspace of the space $C(\mathbb{T} \times \mathbb{T})$ of $2 \pi$-periodic (in $\theta$ and $\varphi$ ) functions on $\mathbb{R}^{2}$ by setting

$$
{ }_{s} V_{j}\left(\mathbb{T}^{2}\right):=\left\{\sum_{k=0}^{2^{j}-1} \sum_{l=0}^{2^{j}-1} C_{k, l} \Phi_{s}^{j, k}(\theta) \Phi_{s}^{j, l}(\varphi): C_{k, l} \in \mathbb{R} \text { for all } k, l=\overline{0,2^{j}-1}\right\} .
$$

The interpolation projection of any function $F(\theta, \varphi) \in C(\mathbb{T} \times \mathbb{T})$ is defined as follows:

$$
S_{s, 2^{j}} F(\theta, \varphi)=P_{s}^{\text {int } V_{j}\left(\mathbb{T}^{2}\right)} F(\theta, \varphi):=\sum_{k=0}^{2^{j}-1} \sum_{l=0}^{2^{j}-1} F\left(\frac{2 \pi k}{2^{j}}, \frac{2 \pi l}{2^{j}}\right) \Phi_{s}^{j, k, l}(\theta, \varphi) .
$$


Obviously, $\left|S_{2^{j}} F(\theta, \varphi)\right| \leq L_{2^{j}}(\theta, \varphi)\|F\|_{C(\mathbb{T} \times \mathbb{T})}$, where $L_{2^{j}}(\theta, \varphi)$ is the Lebesgue function of the operator $S_{2^{j}}: C(\mathbb{T} \times \mathbb{T}) \rightarrow{ }_{s} V_{j} \subset C(\mathbb{T} \times \mathbb{T})$,

$$
L_{s, 2^{j}}(\theta, \varphi)=\sum_{k=0}^{2^{j}-1} \sum_{l=0}^{2^{j}-1}\left|\Phi_{s}^{j, k}(\theta)\right|\left|\Phi_{s}^{j, l}(\varphi)\right|=L_{s, 2^{j}}(\theta) L_{s, 2^{j}}(\varphi),
$$

and $L_{s, 2^{j}}(x)$ is the Lebesgue function of the projection operator of continuous $2 \pi$-periodic functions on the line on the subspace $V_{s}^{j}(\mathbb{T}) \subset C(\mathbb{T})$, which was studied in Lemmas 2 and 3 of the paper [10] under the condition of smoothness of the functions $\widehat{\varphi}_{s}(\omega)$ on $\mathbb{R}$ for $s=2,3$. Using this lemmas and the remark to them on page 265 of the mentioned paper, for the $2 \pi / 2^{j}$-periodic Lebesgue function $L_{s, 2^{j}}(x)$ with $s=2,3$, we obtain

$$
\begin{aligned}
L_{s, 2^{j}}(x) & \leq\left(\bigvee_{1 / 2}^{(1+\varepsilon) / 2}\left(\widehat{\varphi}_{\varepsilon}^{2}(\omega)\right)_{\omega}^{\prime}\left|\frac{\sin 2^{j-1} x}{2^{j-1} x}\right|+\delta_{s, 2} \bigvee_{1 / 2}^{(1+\varepsilon) / 2}\left(\widehat{\varphi}_{\varepsilon}(\omega) \widehat{\varphi}_{\varepsilon}(\omega-1)\right)_{\omega}^{\prime} \frac{\sin ^{2} \varepsilon 2^{j-2} x}{\left|2^{j-1} x\right|}\right) \frac{\left|\sin 2^{j-1} x\right|}{\left|2^{j-1} x\right|}+ \\
+ & {\left[\bigvee_{1 / 2}^{(1+\varepsilon) / 2}\left(\widehat{\varphi}_{\varepsilon}^{2}(\omega)\right)_{\omega}^{\prime}+\delta_{s, 2} \bigvee_{1 / 2}^{(1+\varepsilon) / 2}\left(\widehat{\varphi}_{\varepsilon}(\omega) \widehat{\varphi}_{\varepsilon}(\omega-1)\right)_{\omega}^{\prime}\left(\frac{4}{\pi^{2}}+\varepsilon+\frac{1-4 / \pi^{2}}{2^{2 j}}\right)\left|\sin 2^{j-1} x\right|\right.}
\end{aligned}
$$

for $|x|<2 \pi / 2^{j+1}$. We do not write an analogous estimate for $s=1$, noting only that this estimate is similar to the latter one with replacing $\delta_{s, 2}$ by $\delta_{s, 1}$ and $\widehat{\varphi}_{\varepsilon}(\omega)$ by $\widehat{\varphi}_{1, \varepsilon}(\omega)$ from (1.5).

For brevity, we use the following formulas from [10]:

$$
\Delta^{\varepsilon}=\left[\frac{1}{2}, \frac{1+\varepsilon}{2}\right], \quad \widehat{\varphi}_{3}(\omega)=\widehat{\varphi}_{\varepsilon}^{2}(\omega), \quad \beta(\omega)=\widehat{\varphi}_{\varepsilon}(\omega) \widehat{\varphi}_{\varepsilon}(\omega-1) .
$$

Theorem 1. Assume that, in addition to the conditions ${ }^{2}$ on $\widehat{\varphi}_{s}(\omega)$ imposed in the description of formula (1.4), the functions $\widehat{\varphi}_{3}(\omega)$ and $\beta(\omega)$ are smooth in a neighbourhood of the interval $[(1-\varepsilon) / 2,(1+\varepsilon) / 2]$. Then the Lebesgue constants $L_{s, 2^{j}}(\theta, \varphi), s=2,3$, in (2.2) satisfy on their period $\left[-2 \pi / 2^{j+1}, 2 \pi / 2^{j+1}\right] \times\left[-2 \pi / 2^{j+1}, 2 \pi / 2^{j+1}\right]$ the estimates

$$
\begin{aligned}
L_{s, 2^{j}}(\theta, \varphi) & \leq\left\{\left[\bigvee_{\Delta^{\varepsilon}} \widehat{\varphi}_{3}^{\prime}(\omega)\left|\frac{\sin 2^{j-1} \varphi}{2^{j-1} \varphi}\right|+\delta_{s, 2} \bigvee_{\Delta^{\varepsilon}} \beta^{\prime}(\omega) \frac{\sin ^{2}\left(\varepsilon 2^{j-1} \varphi / 2\right)}{\left|2^{j-1} \varphi\right|}\right] \frac{\left|\sin 2^{j-1} \varphi\right|}{\left|2^{j-1} \varphi\right|}+\right. \\
& \left.+\left[\bigvee_{\Delta^{\varepsilon}} \widehat{\varphi}_{3}^{\prime}(\omega)+\delta_{s, 2} \bigvee_{\Delta^{\varepsilon}} \beta_{3}^{\prime}(\omega)\right]\left(\frac{4}{\pi^{2}}+\frac{1-4 / \pi^{2}}{2^{2 j}}\right)\left|\sin 2^{j-1} \varphi\right|\right\} \times \\
\times & \left\{\left[\bigvee_{\Delta^{\varepsilon}} \widehat{\varphi}_{3}^{\prime}(\omega) \frac{\left|\sin 2^{j-1} \theta\right|}{2^{j-1} \theta}+\delta_{s, 2} \bigvee_{\Delta^{\varepsilon}} \beta^{\prime}(\omega) \frac{\sin ^{2}\left(\varepsilon 2^{j-1} \theta / 2\right)}{\left|2^{j-1} \theta\right|}\right] \frac{\left|\sin 2^{j-1} \theta\right|}{\left|2^{j-1} \theta\right|}+\right. \\
& \left.+\left[\bigvee_{\Delta^{\varepsilon}} \widehat{\varphi}_{3}^{\prime}(\omega)+\delta_{s, 2} \bigvee_{\Delta^{\varepsilon}} \beta^{\prime}(\omega)\right]\left(\frac{4}{\pi^{2}}+\frac{1-4 / \pi^{2}}{2^{2 j}}\right)\left|\sin 2^{j-1} \theta\right|\right\} .
\end{aligned}
$$

P r o o f follows from the above estimate and (2.2).

We note that, to estimate the function $(2.2)$ on the square $\mathbb{T} \times \mathbb{T}$, it is needed to write its estimate on every small square $\left[2 \pi(2 k-1) / 2^{j+1}, 2 \pi(2 k+1) / 2^{j+1}\right] \times\left[2 \pi(2 l-1) / 2^{j+1}, 2 \pi(2 l+1) / 2^{j+1}\right]$ contained in $\mathbb{T} \times \mathbb{T}$ by replacing on the right-hand-side of $(2.3) \varphi$ by $\left(\varphi-2 \pi k / 2^{j}\right)$ and $\theta$ by $\left(\theta-2 \pi l / 2^{j}\right)$.

\footnotetext{
${ }^{2}$ Actually, this is a condition to estimate $L_{s, 2^{j}}(x)$ in [10] allowing to drop terms outside the integrals when integrating by parts.
} 
To estimate the Lebesgue constant, which is the norm in $C(\mathbb{T} \times \mathbb{T})$ of the function $L_{s, 2^{j}}(\theta, \varphi)$ coinciding with the norm of the operator $\left\|S_{2^{j}}\right\|=\left\|S_{2^{j}}\right\|_{C(\mathbb{T} \times \mathbb{T})}^{C(\mathbb{T} \times \mathbb{T})}$, it is sufficient to estimate it on any period, in particular, for $|\theta|<\pi / 2^{j}$ and $|\varphi|<\pi / 2^{j}$. Estimating the right-hand side of (2.3) with the use of the fact that $|\sin x| /|x| \leq 1$ for $|x|<\pi / 2$, we obtain the following result.

Corollary 1. Assume that the conditions of Theorem 1 are satisfied. Then the norm of the operators of the interpolation projection (2.1) from $C(\mathbb{T} \times \mathbb{T})$ to the subspace ${ }_{s} V_{j}\left(\mathbb{T}^{2}\right) \subset C(\mathbb{T} \times \mathbb{T})$ satisfies the estimate

$$
\left\|S_{s, 2^{j}}\right\| \leq\left(\bigvee_{\Delta^{\varepsilon}} \widehat{\varphi}_{3}^{\prime}(\omega)+\delta_{s, 2} \bigvee_{\Delta^{\varepsilon}} \beta^{\prime}(\omega)\right)^{2}\left(\frac{4}{\pi^{2}}+\varepsilon+\frac{1-4 / \pi^{2}}{2^{2 j}}\right)^{2}
$$

For one-dimensional periodic wavelets, the following well-known and easily verified remarkable fact holds: for any $\varepsilon \in(0,1 / 3]$, the operator of interpolation (and also orthogonal) projection on the subspaces $V_{j}$ of periodic multiresolution analysis generated by any Meyer type function $\widehat{\varphi}_{\varepsilon}(\omega)$ is the identity operator on the subspace of trigonometric polynomials of order $N_{\varepsilon}=\left[2^{j-1}(1-\varepsilon)\right]$, where $[a]$ is the integer part of $a$ for $a \geq 0$.

We verify in what form this property is preserved for the operators (2.1). Computing $S_{s, 2^{j}} g(\theta, \varphi)$ for $g(\theta, \varphi)=e^{i \mu \theta} e^{i \eta \varphi}$ and integer $\mu$ and $\eta$, we have

$$
\begin{gathered}
S_{s, 2^{j}} g(\theta, \varphi)=\sum_{k=0}^{2^{j}-1} \sum_{l=0}^{2^{j}-1} e^{2 \pi i \mu k / 2^{j}} e^{2 \pi i \eta l / 2^{j}} \Phi_{s}^{j, k, l}(\theta, \varphi)= \\
=\sum_{\nu} 2^{-j} \widehat{\varphi}_{s}\left(\frac{\nu}{2^{j}}\right) e^{i \nu \theta} \sum_{k=0}^{2^{j}-1} e^{2 \pi i(\mu-\nu) k / 2^{j}} \sum_{\nu^{\prime}} 2^{-j} \widehat{\varphi}_{s}\left(\frac{\nu^{\prime}}{2^{j}}\right) e^{i \nu^{\prime} \varphi} \sum_{l=0}^{2^{j}-1} e^{2 \pi i\left(\eta-\nu^{\prime}\right) l / 2^{j}}= \\
=\sum_{\nu} 2^{-j} \widehat{\varphi}_{s}\left(\frac{\nu}{2^{j}}\right) e^{i \nu \theta} \frac{e^{2 \pi i(\mu-\nu)}-1}{e^{2 \pi i(\mu-\nu) / 2^{j}}-1} \sum_{\nu^{\prime}} 2^{-j} \widehat{\varphi}_{s}\left(\frac{\nu^{\prime}}{2^{j}}\right) e^{i \nu^{\prime} \varphi} \frac{e^{2 \pi i\left(\eta-\nu^{\prime}\right)}-1}{e^{2 \pi i\left(\eta-\nu^{\prime}\right) / 2^{j}}-1}= \\
=\sum_{\nu} 2^{-j} \widehat{\varphi}_{s}\left(\frac{\nu}{2^{j}}\right) e^{i \nu \theta} 2^{j} \delta_{\mu, \nu} \sum_{\nu^{\prime}}^{\left(\mu-\nu^{\prime}\right) / 2^{j}} 2^{-j} \widehat{\varphi}_{s}\left(\frac{\nu^{\prime}}{2^{j}}\right) e^{i \nu^{\prime} \varphi} 2^{j} \delta_{\nu^{\prime}, \eta}=e^{i \mu \theta} e^{i \eta \varphi} \widehat{\varphi}_{s}\left(\frac{\mu}{2^{j}}\right) \widehat{\varphi}_{s}\left(\frac{\eta}{2^{j}}\right),
\end{gathered}
$$

which coincides with $g(\theta, \varphi)$ for $|\mu| / 2^{j} \leq(1-\varepsilon) / 2$ and $|\eta| / 2^{j} \leq(1-\varepsilon) / 2$ (where $\left.\widehat{\varphi}_{s}(\omega) \equiv 1\right)$.

Thus, we obtain the following property of interpolation projections on the subspaces ${ }_{s} V_{j}\left(\mathbb{T}^{2}\right)$.

Assertion 1. For the trigonometric polynomials of two variables

$$
t_{n, m}(\theta, \varphi)=\sum_{\mu=-n}^{n} \sum_{\eta=-m}^{m} a_{\mu, \nu} e^{i(\mu \theta+\nu \varphi)}
$$

of order $n$ in the variable $\theta$ and order $m$ in the variable $\varphi$, the equalities

$$
S_{s, 2^{j}} t_{n, m}(\theta, \varphi) \equiv t_{n, m}(\theta, \varphi)
$$

hold for $n$ and $m$ not greater than $N_{\varepsilon, j}=\left[2^{j-1}(1-\varepsilon)\right]$ and $s=1,2,3$.

Note that the order $N_{\varepsilon, j}$ of the polynomials in (2.5) is allowed in each of the variables $\theta$ and $\varphi$, not just in the totality of variables (when the summation in the formula for $t_{n, m}(\theta, \varphi)$ is taken over $\mu$ and $\nu$ such that $\left.|\mu|+|\nu| \leq N_{\varepsilon, j}\right)$. 
According to the usual Lebesgue scheme, from inequality (2.4) and Assertion 1, we easily obtain an estimate of the error of approximation of continuous $2 \pi$-periodic functions of two variables by their interpolation projections on ${ }_{s} V_{j}\left(\mathbb{T}^{2}\right)$. In view of the importance of this estimate for practical applications of interpolating wavelets, we state it as a theorem. We denote by $E_{n}(F)_{C(\mathbb{T} \times \mathbb{T})}$ the best approximation in the metric of $C(\mathbb{T} \times \mathbb{T})$ of continuous $2 \pi$-periodic functions $F$ on the square $\mathbb{T} \times \mathbb{T}$ by trigonometric polynomials of order $n$ in each variable.

Theorem 2. Under the conditions of Theorem 1 on $\widehat{\varphi_{s}}(\omega), s=2,3$, any function $F(\theta, \varphi)$ in $C(\mathbb{T} \times \mathbb{T})$ satisfies the estimates

$$
\left\|F(\theta, \varphi)-S_{s, 2^{j}} F(\theta, \varphi)\right\|_{C(\mathbb{T} \times \mathbb{T})} \leq\left(1+\left\|S_{s, 2^{j}}\right\|\right) E_{N_{\varepsilon, j}}(F)_{C(\mathbb{T} \times \mathbb{T})} .
$$

P r o o f. To justify this estimate, we note that, applying formula (2.5) to the polynomial $t_{N_{\varepsilon, j}}$ of the best approximation of the function $F$ in $C(\mathbb{T} \times \mathbb{T})$, we obtain

$$
\left\|F(\theta, \varphi)-S_{s, 2^{j}} F(\theta, \varphi)\right\|=\left\|\left(F(\theta, \varphi)-t_{N_{\varepsilon, j}}(\theta, \varphi)\right)+S_{s, 2^{j}}\left(t_{N_{\varepsilon, j}}(\theta, \varphi)-F(\theta, \varphi)\right)\right\|,
$$

From this, using the triangle inequality for norms, the definition of $\left\|S_{s, 2^{j}}\right\|$, and Corollary 1 , we get $(2.6)$.

An estimate of the best approximations $E_{n}(F)_{C(\mathbb{T} \times \mathbb{T})}$ of the Jackson type in terms of the modules of continuity or the parameters $K$ and $\alpha$ of the Hölder class

$$
K H^{\alpha}=\left\{f:|f(x+\Delta x)-f(x)| \leq K|\Delta x|^{\alpha}\right\}
$$

can be found in the known monographs on approximation theory.

The systems of functions

$$
\left\{\Phi_{s}^{j+1,2 k+1,2 l+1}(\theta, \varphi): k=\overline{0,2^{j}-1}\right\}, j \in \mathbb{Z}_{+}(s=1,2,3),
$$

additional to (1.7) are the interpolation bases of the subspaces ${ }_{s} W_{j}\left(\mathbb{T}^{2}\right)\left({ }_{s} V_{j+1}\left(\mathbb{T}^{2}\right)={ }_{s} V_{j}\left(\mathbb{T}^{2}\right) \oplus_{s}\right.$ $\left.W_{j}\left(\mathbb{T}^{2}\right), j \in \mathbb{Z}_{+}\right)$. By their means, any function $g \in_{s} V_{j+1}\left(\mathbb{T}^{2}\right)$ is uniquely represented in the form

$$
g=P_{s}^{i n t} g+P_{s W_{j}}^{i n t}\left(g-P_{s}^{i n t} g\right),
$$

which is easily derived from the fact that ${ }_{s} W_{j} \subset{ }_{s} V_{j+1}$. For each $s=1,2,3$, the family of systems (2.7) over all $j \in \mathbb{Z}_{+}$together with $\Phi_{0,0} \equiv 1$ is an interpolation basis of the whole space $C(\mathbb{T} \times \mathbb{T})$, so that any function $F(\theta, \varphi)$ is expanded in the series

$$
F(\theta, \varphi)=F(0,0)+\sum_{j=0}^{\infty} \sum_{k, l=0}^{2^{j}-1} c_{j, k, l} \Phi_{s}^{j+1,2 k+1,2 l+1}(\theta, \varphi)
$$

converging uniformly in the square $\mathbb{T} \times \mathbb{T}$ and, hence, in $\mathbb{R}^{2}$. According to the usual scheme, the coefficients of this series are calculated recursively in $j$ in terms of the grid values of the function $F$ and the partial sums

$$
\Sigma_{j-1}(\theta, \varphi ; F)=F(0,0)+\sum_{\lambda=0}^{j-1} \sum_{\mu, \nu=0}^{2^{\lambda}-1} c_{\lambda, \mu, \nu} \Phi_{s}^{\lambda+1,2 \mu+1,2 \nu+1}(\theta, \varphi)
$$

of the same series, namely

$$
c_{j, k, l}=F\left(\frac{2 \pi(2 k+1)}{2^{j+1}}, \frac{2 \pi(2 l+1)}{2^{j+1}}\right)-\Sigma_{j-1}\left(\frac{2 \pi(2 k+1)}{2^{j+1}}, \frac{2 \pi(2 l+1)}{2^{j+1}}\right) .
$$


It follows from (2.8) that the sum $\Sigma_{j-1}(\theta, \varphi ; F)$ coincides with $P_{s}^{i n t} F(\theta, \varphi)=S_{s, 2^{j}} F(\theta, \varphi)$ (see (2.1)), so that we can write the values $c_{j, k, l}$ without recurrence:

$$
c_{j, k, l}=\left(F-P_{s}^{\operatorname{int}} F\right)\left(\frac{2 \pi(2 k+1)}{2^{j+1}}, \frac{2 \pi(2 l+1)}{2^{j+1}}\right) .
$$

Hence, it is easily deduced that the series (2.9) with the coefficients (2.11) coincides with the series

$$
F(\theta, \varphi)=F(0,0)+\sum_{j=0}^{\infty}\left(S_{s, 2^{j+1}} F(\theta, \varphi)-S_{s, 2^{j}} F(\theta, \varphi)\right)
$$

converging uniformly in $\mathbb{T} \times \mathbb{T}$ by Theorem 2 . The partial sum of order $J$ of the latter series is

$$
F(0,0)+\sum_{j=0}^{J-1}\left(S_{s, 2^{j+1}}-S_{s, 2^{j}}\right) F(\theta, \varphi)=S_{s, 2^{J}} F(\theta, \varphi)=\Sigma_{J-1}(\theta, \varphi ; F) .
$$

\section{Interpolating wavelets on the sphere and their application to the approximation of functions in $C(\mathbb{S})$}

In the second section, unlike the first section, the $\operatorname{arguments}(\theta, \varphi)$ of the functions $F$ and $\Phi_{s}^{j, k, l}$ were treated as the Cartesian coordinates of points of the square $\mathbb{T} \times \mathbb{T}$ on the opposite sides of which the values of any function in $C(\mathbb{T} \times \mathbb{T})$ coincide in view of its $2 \pi$-periodicity. Moreover, the function $F(\theta, \varphi)$ constructed on $\widetilde{\mathbb{S}}_{(2)}$ by formula (1.1), if interpreted as a function on the square $\mathbb{T} \times \mathbb{T}$, has the additional feature that it is constant on each of the sides $\theta=0$ and $\theta=\pi$ of the square.

Let $F$ be a function defined on the sphere $\mathbb{S}$ and continuously depending on the points of the sphere. For instance, $F$ represented as $F\left(x_{1}, x_{2}, x_{3}\right)$ is a function continuous in all coordinates connected by the relation $x_{1}^{2}+x_{2}^{2}+x_{3}^{2}=1$. In particular, $F$ is also continuous at the poles $N$ and $S$ of the sphere $\mathbb{S}$. Therefore, after the change $x_{1}=\cos \varphi \sin \theta, x_{2}=\cos \varphi \cos \theta, x_{3}=\sin \theta$, the function $F$ becomes a function of the coordinates $\varphi \in \mathbb{T}=[0,2 \pi)$ and $\theta \in[0, \pi]$ with the following specificity: $F(N)$ and $F(S)$ do not depend on $\theta$, since $\lim _{\theta \rightarrow 0} F(\theta, \varphi)=F(N)$ and $\lim _{\theta \rightarrow \pi} F(\theta, \varphi)=F(S)$ for any $\varphi \in[0,2 \pi]$. Thus, the function $F(\theta, \varphi)$ defined on the double sphere $\widetilde{\mathbb{S}}_{(2)}$ by formula (1.1) and glued from the continuous functions $f(\theta, \varphi)$ on $M_{\varphi}$ for $\theta \uparrow_{0}^{\pi}$ and $f(2 \pi-\theta, \varphi \pm \pi)$ on $M_{\varphi \pm \pi}$ for $\theta \uparrow_{\pi}^{2 \pi}$ is continuous on $\widetilde{\mathbb{S}}_{(2)}$, since the values of the function $F(0, \varphi)$ and the values of the function $F(\pi, \varphi)$ do not depend on $\varphi$ at the gluing points $\theta=2 \pi$ and $\theta=\pi$.

We note that the values of the function $F(\theta, \varphi)$ on $\mathbb{S}$ (i.e., for $\theta \uparrow_{0}^{\pi}, \varphi \uparrow_{0}^{2 \pi}$ ) coincide with the values of the original function $f(\theta, \varphi)$. Therefore, approximating $F$ on $\widetilde{\mathbb{S}}_{(2)}$, we simultaneously approximate $f$ on $\mathbb{S}$. Of course, the latter property could be preserved for any continuous extension of $f$ from $\mathbb{S}$ to $\widetilde{\mathbb{S}}_{(2)} \backslash \mathbb{S}$. However, if the original function $f$ is smooth on $\mathbb{S}$, i.e., at any point $\left(x_{1}, x_{2}, x_{3}, f\left(x_{1}, x_{2}, x_{3}\right)\right.$ ) (with $x_{1}^{2}+x_{2}^{2}+x_{3}^{2}=1$ ) of the graph surface of $f$ over $\mathbb{S}$, there exists a tangent plane to the graph, then, obviously, the extension chosen by means of (1.4) preserves the smoothness of $F(\theta, \varphi)$ on any full $\varphi$-meridian $C_{\varphi}$ and, hence, on the whole double sphere $\widetilde{\mathbb{S}}_{(2)}$, since there exists a tangent line to the graph of $F(\theta, \varphi)$ over any full $\varphi$-meridian at the points $(\theta, \varphi)\left(\theta \uparrow \begin{array}{l}\pi \\ 0\end{array}\right)$ which is the section of the tangent plane at the point $X(\theta, \varphi) \in C_{\varphi}$ by the plane containing $C_{\varphi}$.

The basis functions $\Phi_{s}^{j, k, l}(\theta, \varphi)$ are defined on the whole $\widetilde{\mathbb{S}}_{(2)}$ as $2 \pi$-periodic in $\theta$ and $\varphi$, since the parameter $\theta$ changes from 0 to $2 \pi$ on any full $\varphi$-meridian and the parameter $\varphi$ defining $C_{\varphi}$ changes similarly. Of course, not each of these functions is constant for $\theta=0(\theta=2 \pi)$ or $\theta=\pi$ like $F(\theta, \varphi)$ (these are the functions $\Phi_{s}^{j, 0, l}(\theta, \varphi)$ and $\Phi_{s}^{j, 2^{j-1}, l}(\theta, \varphi)$ ). However, only the continuity of $F$ is important to apply formula (2.1), estimates (2.3), (2.4), and (2.6), and formulas (2.9)-(2.12) to 
the functions $F(\theta, \varphi)$ defined by (1.1). Thus, the functions $\Phi_{s}^{j, k, l}(\theta, \varphi)$ defined by (1.7) determine multiresolution analysis on $\widetilde{\mathbb{S}}_{(2)}$, i.e. the subspaces ${ }_{s} V_{j}\left(\widetilde{\mathbb{S}}_{(2)}\right)$ and ${ }_{s} W_{j}\left(\widetilde{\mathbb{S}}_{(2)}\right)$. In so doing, a pair $(\theta, \varphi)$ should be treated everywhere as parameters defining the points $M(\theta, \varphi)$ on $\widetilde{\mathbb{S}}_{(2)}$. The only useful thing remaining is to rewrite formulas $(2.1)$ in terms of the function $f(\theta, \varphi)$ on $\mathbb{S}$ defining $F(\theta, \varphi)$ on $\widetilde{\mathbb{S}}_{(2)}$. Using $(2.1)$ and $(1.1)$, we set

$$
\begin{gathered}
P_{s V_{j}}^{i n t} F(\theta, \varphi)=\sum_{k=0}^{2^{j-1}-1} \sum_{l=0}^{2^{j}-1} f\left(\frac{2 \pi k}{2^{j}}, \frac{2 \pi l}{2^{j}}\right) \Phi_{s}^{j, k, l}(\theta, \varphi)+ \\
+\sum_{k=2^{j-1}}^{2^{j}-1}\left(\sum_{l=0}^{2^{j-1}-1} f\left(\frac{2 \pi\left(2^{j}-k\right)}{2^{j}}, \frac{2 \pi\left(l+2^{j-1}\right)}{2^{j}}\right) \Phi_{s}^{j, k, l}(\theta, \varphi)+\sum_{l=2^{j-1}}^{2^{j}-1} f\left(\frac{2 \pi\left(2^{j}-k\right)}{2^{j}}, \frac{2 \pi l}{2^{j}}\right) \Phi_{s}^{j, k, l}(\theta, \varphi)\right) .
\end{gathered}
$$

By Theorem 2, one can estimate the error of approximation of the function $F(\theta, \varphi)$ by means of $P_{s V_{j}}^{i n t} F(\theta, \varphi)$ in terms of the best approximation $E_{N_{\varepsilon, l}}(F)_{C\left(\widetilde{\mathbb{S}}_{(2)}\right)}$. In real applied problems, it is unlikely to be required to approximate functions defined on both inner and outer sides of the sphere $\mathbb{S}$. Therefore, to approximate the original function $f(\theta, \varphi)$, it is sufficient to estimate the deviation $\left|f(\theta, \varphi)-P_{s V_{j}}^{i n t} F(\theta, \varphi)\right|_{C([0, \pi] \times \mathbb{T})}$ that does not exceed the approximation error (2.6).

There are studies of the problem of approximation by trigonometric polynomials on an interval less than the period. Here, one can expect an essential improvement of the estimate (2.6) by learning to use the specificity of the function $f$ on $\mathbb{S}$, in particular, its singularities on $\mathbb{S}$ which make it hardly changing in a neighborhood of the sides of the rectangle $[0, \pi] \times \mathbb{T}$ with $\theta=0$ and $\theta=\pi$ on which the function $f(\theta, \varphi)$ is naturally transferred from $\mathbb{S}$.

Until now, we have discussed the use of interpolation properties of the wavelets $\Phi_{s}^{j, k, l}(\theta, \varphi)$. As noted, in view of (1.6) the systems $\left\{2^{j / 2} \Phi_{s}^{j, k}(x): k=\overline{0,2^{j}-1}\right\}$ are orthonormal for $s=1$ or $s=2$ and for every $j \in \mathbb{N}$. This implies that, for any $j \in \mathbb{N}$, the systems $\left\{2^{j / 2} \Phi_{2}^{j, k, l}(\theta, \varphi): k, l=\overline{0,2^{j}-1}\right\}$ are also orthonormal:

$$
\begin{aligned}
\left(\frac{1}{2 \pi}\right)^{2} \int_{0}^{2 \pi} \int_{0}^{2 \pi} 2^{j / 2} \Phi_{2}^{j, k, l}(\theta, \varphi) 2^{j / 2} \overline{\Phi_{2}^{j, m, n}(\theta, \varphi)} d \theta d \varphi=\frac{1}{2 \pi} \int_{0}^{2 \pi} 2^{j / 2} \Phi_{2}^{j, k}(\theta) \overline{\Phi_{2}^{j, m}(\theta)} d \theta \times \\
\times \frac{1}{2 \pi} \int_{0}^{2 \pi} 2^{j / 2} \Phi_{2}^{j, l}(\varphi) \overline{\Phi_{2}^{j, n}(\varphi)} d \varphi=\delta_{k, m} \cdot \delta_{l, n}= \begin{cases}1, & (k, l)=(m, n), \\
0, & (k, l) \neq(m, n) .\end{cases}
\end{aligned}
$$

However, the question on the application of the orthonormality properties of these systems to the approximation of functions on the sphere in $L^{2}(\mathbb{S})$-norm requires separate consideration.

\section{Conclusion}

In this paper, we have considered the question of approximation of continuous functions on the sphere $\mathbb{S} \subset \mathbb{R}^{3}$ and have proposed once more approach to the construction of corresponding interpolating wavelets. Due to a special double expansion of the sphere, this approach reduces to the simple and well-studied problem on the construction of interpolating periodic wavelets on the plane $\mathbb{R}^{2}$. Two of the constructed wavelet systems are orthogonal on the expanded sphere $\mathbb{S}$. This property can be useful when the approximated function is inaccurately defined (for instance, is obtained experimentally). The problem of accuracy of approximation of functions on the sphere in $L^{2}$ was not studied in this paper.

\section{REFERENCES}

1. Arfaoui S., Rezgui I., Mabrouk A.B. Wavelet Analysis on the Sphere: Spheroidal Wavelets. Berlin: Walter de Gruyter GmbH \& Co KG, 2017. 144 p. 
2. Askari-Hemmat A., Dehghan M. A., Skopina M. Polynomial Wavelet-Type Expansions on the Sphere. Math. Notes, 2003. Vol. 74, No. 2. P. 278-285. DOI: 10.1023/A:1025016510773

3. Chernykh N.I., Subbotin Yu. N. Interpolating-orthogonal wavelet systems. Proc. Steklov Inst. Math., 2009. Vol. 264, Suppl. 1. P. 107-115. DOI: 10.1134/S0081543809050083

4. Dahlke S., Dahmen W., Weinreich I., Schmitt E. Multiresolution analysis and wavelets on $\mathbb{S}^{2}$ and $\mathbb{S}^{3}$. Numer. Funct. Anal. Optim., 1995. Vol. 16, No. 1-2. P. 19-41. DOI: 10.1080/01630569508816605

5. Dai F. Characterizations of function spaces on the sphere using frames. Trans. Amer. Math. Soc., 2007. Vol. 359, No. 2. P. 567-589. DOI: 10.1090/S0002-9947-06-04030-X

6. Farkov Yu. B-spline wavelets on the sphere. In: Proc. of the Intern. Workshop "Self-Similar Systems", 1999. Vol. 30. P. 79-82.

7. Freeden W., Schreiner M. Orthogonal and nonorthogonal multiresolution analysis, scale discrete and exact fully discrete wavelet transform on the sphere. Constr. Approx., 1998. Vol. 14, No. 4. P. $493-515$. DOI: $10.1007 / \mathrm{s} 003659900087$

8. Schröder P., Sweldens W. Spherical wavelets: Efficiently representing functions on the sphere. In: Wavelets in the Geosciences. Lect. Notes in Earth Sci., vol. 90. 1995. P. 158-188. DOI: $10.1007 / \mathrm{BFb0011096}$

9. Skopina M. Polynomial Expansions of Continuous Functions on the Sphere and on the Disk. IMI Research Reports, Department of Mathematics, University of South Carolina, 2001. Preprint, Vol. 5.13 p. http://imi.cas.sc.edu/django/site_media/media/papers/2001/2001_05.pdf

10. Subbotin Yu. N., Chernykh N. I. Interpolation wavelets in boundary value problems. Proc. Steklov Inst. Math., 2018. Vol. 300, Suppl. 1. P. 172-183. DOI: 10.1134/S0081543818020177 